\title{
Umsetzung von Aminoarsinen mit tri- und tetrafunktionellen Alkoholen
}

\author{
Reaction of Aminoarsines with Tri- and Tetrafunctional Alcoholes \\ Werner J. RühL und Friedhelm Kober \\ Fachbereich 8 der Technischen Hochschule Darmstadt, E. Zintl-Institut \\ (Z. Naturforsch. 31 b, 307-310 [1976]; eingegangen am 18. August/26. November 1975) \\ Arsinous Acid Esters, Aminoarsines
}

\begin{abstract}
The reactions of the tri- and tetrafunctional alcoholes $\mathrm{CH}_{3} \mathrm{C}\left(\mathrm{CH}_{2} \mathrm{OH}\right)_{3}$ and $\mathrm{C}\left(\mathrm{CH}_{2} \mathrm{OH}\right)_{4}$ with the aminoarsines $\left(\mathrm{CH}_{3}\right)_{2} \mathrm{As}-\mathrm{N}\left(\mathrm{CH}_{3}\right)_{2}, \mathrm{CH}_{3} \mathrm{As}\left[\mathrm{N}\left(\mathrm{CH}_{3}\right)_{2}\right]_{2}$, and $\mathrm{As}\left[\mathrm{N}\left(\mathrm{CH}_{3}\right)_{2}\right]_{3}$ are examined. The reactions lead by cleavage of the As-N-bond to the formation of acyclic and mono- and bicyclic arsinous acid esters.
\end{abstract}

\section{Einleitung}

Arsinigsäureester des Pentaerythrits $\mathrm{C}\left(\mathrm{CH}_{2} \mathrm{OH}\right)_{4}$ und des 1.1.1-Tris-(hydroxymethyl)-äthans $\mathrm{CH}_{3} \mathrm{C}\left(\mathrm{CH}_{2} \mathrm{OH}\right)_{3}$ wurden oft bearbeitet ${ }^{1-7}$. Im Vordergrund des Interesses stand die noch nicht eindeutig geklärte Frage, ob der Arsinigsäure-Ester des Pentaerythrits in der Form (A) oder (B) vorliegt.

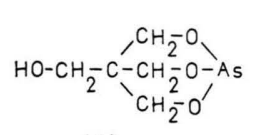

(A)

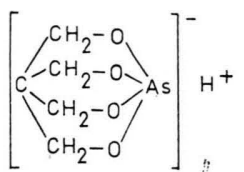

(B)

Im Zusammenhang mit Arbeiten über cyclische Arsinigsäureester ${ }^{8,9}$ haben wir dieses Problem aufgegriffen und berichten in der vorliegenden Arbeit über Umsetzungen von Dimethylaminodimethylarsin, Bis-(dimethylamino)-methylarsin und Tris(dimethylamino)-arsin mit Pentaerythrit und 1.1.1Tris-(hydroxymethyl)-äthan.

Sonderdruckanforderungen an Dr. FrIEdHelm KoBer, Fachbereich 8 der Technischen Hochschule Darmstadt, E. Zintl-Institut, D-6100 Darmstadt, Hochschulstraße 4, BRD.

\section{Allgemeiner Teil}

Umsetzungen mit $\left(\mathrm{CH}_{3}\right)_{2} \mathrm{As}-\mathrm{N}\left(\mathrm{CH}_{3}\right)_{2}$

Pentaerythrit setzt sich mit Dimethylaminodimethylarsin im Molverhältnis $1: 4$ nach Gl. (1) zum Tetrakis-O-dimethylarsinopentaerythrit (1) um.

$$
\begin{gathered}
\mathrm{C}\left(\mathrm{CH}_{2} \mathrm{OH}\right)_{4}+4\left(\mathrm{CH}_{3}\right)_{2} \mathrm{As}-\mathrm{N}\left(\mathrm{CH}_{3}\right)_{2} \\
\mathrm{C}\left[\mathrm{CH}_{2} \mathrm{OAs}\left(\mathrm{CH}_{3}\right)_{2}\right]_{4}+4 \mathrm{HN}\left(\mathrm{CH}_{3}\right)_{2} \\
\mathbf{1}
\end{gathered}
$$

Mit drei, zwei oder einem Äquivalent

$$
\left(\mathrm{CH}_{3}\right)_{2} \mathrm{As}-\mathrm{N}\left(\mathrm{CH}_{3}\right)_{2}
$$

führt die Umsetzung nicht zu den erwarteten tri-, di- oder monokakodylsubstituierten Estern, sondern zu destillativ nicht-trennbaren Gemischen von $\left(\mathrm{HOCH}_{2}\right)_{m} \mathrm{C}\left[\mathrm{CH}_{2} \mathrm{OAs}\left(\mathrm{CH}_{3}\right)_{2}\right]_{n} \quad$ (mit $\left.m+n=4\right)$. Durch Zusatz von $\left(\mathrm{CH}_{3}\right)_{2} \mathrm{As}-\mathrm{N}\left(\mathrm{CH}_{3}\right)_{2}$ tritt Umsetzung dieses Gemisches zu C[CH $\left.\mathrm{CH}_{2} \mathrm{OAs}\left(\mathrm{CH}_{3}\right)_{2}\right]_{4}$ ein.

Ähnliche Ergebnisse fanden wir beim 1.1.1-Tris(hydroxymethyl)-äthan. $\mathrm{Mit}\left(\mathrm{CH}_{3}\right)_{2} \mathrm{As}-\mathrm{N}\left(\mathrm{CH}_{3}\right)_{2}$ tritt im Molverhältnis 1:3 Umsetzung (2) zum 1.1.1-Tris(dimethylarsinooxymethyl)-äthan (2) ein.

$$
\begin{aligned}
& \mathrm{CH}_{3}-\mathrm{C}\left(\mathrm{CH}_{2} \mathrm{OH}\right)_{3}+3\left(\mathrm{CH}_{3}\right)_{2} \mathrm{As}-\mathrm{N}\left(\mathrm{CH}_{3}\right)_{2} \\
& \mathrm{CH}_{3}-\mathrm{C}\left[\mathrm{CH}_{2} \mathrm{OAs}\left(\mathrm{CH}_{3}\right)_{2}\right]_{3}+3 \mathrm{HN}\left(\mathrm{CH}_{3}\right)_{2} \\
& \quad 2
\end{aligned}
$$

Im Molverhältnis 1:2 und 1:1 entsteht nach Gl.(3) 2-Bis-(dimethylarsinooxymethyl)-propanol(1)

(3). Das monosubstituierte

$$
\mathrm{CH}_{3}-\mathrm{C}\left(\mathrm{CH}_{2} \mathrm{OH}\right)_{2}\left[\mathrm{CH}_{2} \mathrm{OAs}\left(\mathrm{CH}_{3}\right)_{2}\right]
$$

kann nicht nachgewiesen werden.

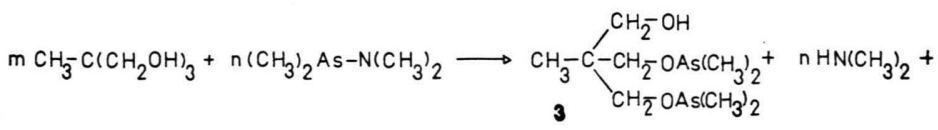

$$
\begin{aligned}
& m=1, n=2 ; \quad m=2, n=2 . \\
& \text { (m-1) } \mathrm{CH}_{3} \mathrm{C}\left(\mathrm{CH}_{2} \mathrm{OH}\right)_{3}
\end{aligned}
$$


Umsetzungen mit $\mathrm{CH}_{3} \mathrm{As}\left[\mathrm{N}\left(\mathrm{CH}_{3}\right)_{2}\right]_{2}$

Die Umsetzungen mit $\mathrm{CH}_{3} \mathrm{As}\left[\mathrm{N}\left(\mathrm{CH}_{3}\right)_{2}\right]_{2}$ führen nur beim $\mathrm{CH}_{3} \mathrm{C}\left(\mathrm{CH}_{2} \mathrm{OH}\right)_{3}$ im Molverhältnis $1: 1 \mathrm{zu}$ einer nicht-polymeren Verbindung. Nach Gl. (4) entsteht das 5-Hydroxymethyl-2.5-dimethyl-[1.3.2]dioxarsenan.

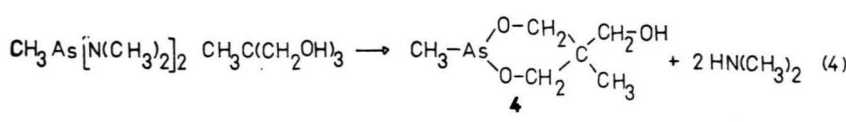

Das IR- und NMR-Spektrum von 4 zeigen eine freie $\mathrm{OH}$-Gruppe durch eine Absorption bei $3400 \mathrm{~cm}^{-1}$ bzw. ein Signal bei $\delta=3,6 \mathrm{ppm}$ an, wobei zusätzlich die Integration der NMR-Signale die
Molekülstruktur 4 bestätigt. Außerdem zeigt eine alkoholische Lösung von $\mathbf{4}$ fast die gleiche Leitfähigkeit wie das reine Lösungsmittel. Somit ist eine Struktur (C) mit Sicherheit auszuschließen.<smiles>C[In]1C[As]2(C)OCC1CO2</smiles>

(C)

Ein weiterer Strukturbeweis für 4 ist die Umsetzung mit $\left(\mathrm{CH}_{3}\right)_{2} \mathrm{As}-\mathrm{N}\left(\mathrm{CH}_{3}\right)_{2}$, die nach Gl. (5) unter Umsetzung der OH-Gruppe zu einem 5Dimethylarsinooxymethyl-2.5-dimethyl-[1.3.2]-dioxarsenan (5) führt.

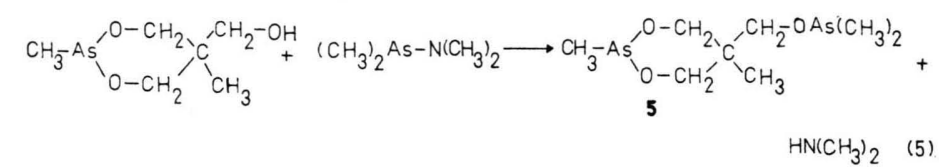

Die Umsetzungen des $\mathrm{C}\left(\mathrm{CH}_{2} \mathrm{OH}\right)_{4}$ mit $\mathrm{CH}_{3} \mathrm{As}\left[\mathrm{N}\left(\mathrm{CH}_{3}\right)_{2}\right]_{2}$ führen zu Verbindungen, deren Analysen-Werte zwar den erwarteten entsprechen. Die hohen Siedepunkte $\left(\sim 200^{\circ} \mathrm{C} / 10^{-2} \mathrm{mmHg}\right)$, die geringe Löslichkeit und Molmassen um 800 weisen aber auf polymere Verbindungen hin.

\section{Umsetzungen mit $\mathrm{As}\left[\mathrm{N}\left(\mathrm{CH}_{3}\right)_{3}\right]_{3}$}

Bei Umsetzung des Tris-(dimethylamino)-arsins mit $\mathrm{C}\left(\mathrm{CH}_{2} \mathrm{OH}\right)_{4}$ werden alle As-N-Bindungen gespalten. Nach Gl. (6a) entsteht das auf anderem Weg schon präparierte 4-Hydroxymethyl-2.6.7trioxa-1-arsa-bicyclo[2.2.2] octan $(6 \mathrm{a})^{3,4,7}$.

Mit $\mathrm{CH}_{3} \mathrm{C}\left(\mathrm{CH}_{2} \mathrm{OH}\right)_{3}$ entsteht nach $\mathrm{Gl}$. $(6 \mathrm{~b})$ das ebenfalls schon bekannte 4-Methyl-2.6.7-trioxa-1arsa-bicyclo[2.2.2]octan $(6 \mathrm{~b})^{3,4,7}$.

$$
\begin{aligned}
& \text { X-C(CH}{ }_{2} \mathrm{OH}_{3}+\mathrm{As}\left[{\left.\mathrm{N}\left(\mathrm{CH}_{3}\right)_{2}\right]_{3}}^{\rightarrow} \underset{\mathrm{X}}{\text { 6a: } \mathrm{X}=-\mathrm{CH}_{2} \mathrm{OH}}\right. \\
& \text { 6 b: } \mathrm{X}=-\mathrm{CH}_{3}
\end{aligned}
$$

Bei 6 a schließen ähnlich wie bei 4 das IR- und ${ }^{1} \mathrm{H}-\mathrm{NMR}$-Spektrum, die eine freie $\mathrm{OH}$-Gruppe zeigen, sowie die geringe Leitfähigkeit in alkoholischer Lösung eine Struktur analog (B) oder (C) aus.

Die Molekülstrukturen wurden durch die Elementaranalysen und die Aufnahme der IR- und ${ }^{1} \mathrm{H}-\mathrm{NMR}$-Spektren sowie die Molekulargewichtsbestimmungen gesichert. Siedepunkte und die Ergebnisse der Analysen und Molekulargewichtsbe- stimmungen sind in Tab. I, IR- und ${ }^{1} \mathrm{H}-\mathrm{NMR}$ Spektren der noch nicht beschriebenen Verbindungen sind in Tab. II zusammengefaßt.

Die IR-Spektren wurden auf einem Modell 337 der Firma Perkin-Elmer als dünne Filme zwischen $\mathrm{KBr}$-Platten gemessen.

Die Spektren bestätigen durch das Auftreten der für die funktionellen Gruppen charakteristischen Signale sowie die Integration der Signale bei den NMR-Spektren die Molekülstrukturen.

Die NMR-Spektren wurden auf einem Gerät T60 der Firma Varian aufgenommen. Angegeben sind in Tab. II die $\delta$-Werte bezogen auf TMS.

Die Molekulargewichte wurden dampfdruckosmometrisch in benzolischer Lösung auf einem Gerät der Firma Knauer bestimmt.

Der C,H-Gehalt wurde durch Verbrennungsanalyse auf einem Gerät Elemental Analyzer der Firma Perkin-Elmer ermittelt.

\section{Experimenteller Teil}

$$
\begin{aligned}
& \text { Die Aminoarsine } \\
& \left(\mathrm{CH}_{3}\right)_{2} \mathrm{As}-\mathrm{N}\left(\mathrm{CH}_{3}\right)_{2}, \mathrm{CH}_{3} \mathrm{As}\left[\mathrm{N}\left(\mathrm{CH}_{3}\right)_{2}\right]_{2} \text { und } \\
& \mathrm{As}\left[\mathrm{N}\left(\mathrm{CH}_{3}\right)_{2}\right]_{3}
\end{aligned}
$$

wurden durch Umsetzung der Chloride $\left(\mathrm{CH}_{3}\right)_{2} \mathrm{AsCl}$, $\mathrm{CH}_{3} \mathrm{AsCl}_{2}$ und $\mathrm{AsCl}_{3}$ mit Dimethylamin in Äther nach den entsprechenden Vorschriften erhalten ${ }^{10-13}$. Bei den Alkoholen wurden käufliche Präparate eingesetzt.

Die Umsetzungen wurden nach einem einheitlichen Verfahren durchgeführt und sollen allgemein beschrieben werden: Die Alkohole werden feingepulvert in abs. Benzol suspendiert und unter heftigem Rühren und Begasen mit trockenem Stick- 
stoff eine benzolische Lösung des Aminoarsins im Verlauf einiger Stunden zugetropft. Die Umsetzung setzt sofort ein und wird nach dem Zutropfen des Aminoarsins durch mehrstündiges Erhitzen unter Rückfluß vervollständigt. Dann wird das Benzol bei Normaldruck und das Produkt bei den in Tab. I angegebenen Bedingungen destilliert.
Wir danken Herrn Prof. Dr. J. Grobe für seine Anregungen und Diskussionen sowie die Unterstützung der Arbeit durch Mittel des Institutes. Der Deutschen Forschungsgemeinschaft danken wir für die finanzielle Unterstützung der Arbeit.

Tab. I. Siedepunkte, Ausbeuten, C,H-Analysen und Molekulargewichtsbestimmungen der Arsinigsäureester.

\begin{tabular}{|c|c|c|c|c|c|}
\hline & Arsinigsäuree & ster & Siedepunkt & C,H-Analyse und (Mol.-Masse) & Ausbeute \\
\hline$(\mathbf{1})$ & $\mathrm{C}_{13} \mathrm{H}_{32} \mathrm{As}_{4} \mathrm{O}_{4}$ & $(538)$ & $128 / 0,5$ & $\begin{array}{l}\text { Ber. C 28,3 H } 5,8 \\
\text { Gef. C 28,5 H } 5,8 \\
\text { (Mol.-Masse 545) }\end{array}$ & 85 \\
\hline (2) & $\mathrm{C}_{11} \mathrm{H}_{27} \mathrm{As}_{3} \mathrm{O}_{3}$ & $(432)$ & $100 / 0,5$ & $\begin{array}{l}\text { Ber. C 30,6 H } 6,2 \\
\text { Gef. C 30,9 H 6,1 } \\
\text { (Mol.-Masse 400) }\end{array}$ & 93 \\
\hline (3) & $\mathrm{C}_{9} \mathrm{H}_{22} \mathrm{As}_{2} \mathrm{O}_{3}$ & $(328)$ & $105 / 0,3$ & $\begin{array}{l}\text { Ber. C } 32,9 \text { H } 6,7 \\
\text { Gef. } \\
\text { (Mol.-Masse } 34,7 \text { H } 6,6\end{array}$ & 60 \\
\hline (4) & $\mathrm{C}_{6} \mathrm{H}_{13} \mathrm{AsO}_{3}$ & $(208)$ & $107 / 0,4$ & $\begin{array}{l}\text { Ber. C } 34,6 \text { H } 6,2 \\
\text { Gef. C 34,9 H 6,2 } \\
\text { (Mol.-Masse 230) }\end{array}$ & 75 \\
\hline (5) & $\mathrm{C}_{8} \mathrm{H}_{18} \mathrm{As}_{2} \mathrm{O}_{3}$ & (312) & $100 / 0,1$ & $\begin{array}{l}\text { Ber. C } 30,8 \text { H } 5,7 \\
\text { Gef. } \\
\text { (Mol.-Masse } 320 \text { ) }\end{array}$ & 80 \\
\hline$(6 \mathbf{a})$ & $\mathrm{C}_{5} \mathrm{H}_{9} \mathrm{AsO}_{4}$ & $(208)$ & $120 / 0,1$ & $\begin{array}{l}\text { Ber. C 28,8 } \text { H 4,3 } \\
\text { Gef. C 28,6 H } 4,3 \\
\text { (Mol.-Masse 230) }\end{array}$ & 75 \\
\hline$(6 \mathrm{~b})$ & $\mathrm{C}_{5} \mathrm{H}_{9} \mathrm{AsO}_{3}$ & $(192)$ & $45 / 0,1$ & $\begin{array}{l}\text { Ber. C 31,2 } \\
\text { Gef. H 4,7 } \\
\text { (Mol.-Masse 205) }\end{array}$ & 90 \\
\hline
\end{tabular}

Tab. II. Spektroskopische Daten.

\begin{tabular}{|c|c|c|}
\hline Ester & ${ }^{1} \mathrm{H}-\mathrm{NMR}$-Spektrum [ppm] & IR-Spektrum $\left[\mathrm{cm}^{-1}\right]$ \\
\hline $\mathbf{1}$ & $\begin{array}{l}\delta\left(\mathrm{AsCH}_{3}\right)=1,2(\mathrm{~s}) \\
\delta\left(\mathrm{OCH}_{2}\right)=3,3(\mathrm{~s}) \\
\text { Integration } 3: 1\end{array}$ & $\begin{array}{l}2990(\mathrm{~s}), 2920(\mathrm{ss}), 2870(\mathrm{~s}), 1480(\mathrm{~s}), 1430(\mathrm{~s}), 1250(\mathrm{~s}), 1160(\mathrm{~m}), \\
1050(\mathrm{ss}), 1020(\mathrm{ss}), 890(\mathrm{~s}), 825(\mathrm{~s}), 750(\mathrm{~m}), 710(\mathrm{~m}), 685(\mathrm{~s}), \\
580(\mathrm{~s}) .\end{array}$ \\
\hline 2 & $\begin{array}{l}\delta\left(\mathrm{AsCH}_{3}\right)=0,7(\mathrm{~s}) \\
\delta\left(\mathrm{OCH}_{2}\right)=3,3(\mathrm{~s}) \\
\delta\left(\mathrm{CH}_{3}\right)=1,2(\mathrm{~s}) \\
\text { Integration } 6: 2: 1\end{array}$ & $\begin{array}{l}3000(\mathrm{ss}), 2930(\mathrm{ss}), 2870(\mathrm{ss}), 1480(\mathrm{~s}), 1440(\mathrm{ss}), 1380(\mathrm{~m}) \\
1260(\mathrm{~s}), 1050(\mathrm{ss}), 1025(\mathrm{ss}), 975(\mathrm{~m}), 890(\mathrm{~s}), 835(\mathrm{~s}), 715(\mathrm{~s}) \\
690(\mathrm{~s}), 590(\mathrm{ss}), 580(\mathrm{ss}) .\end{array}$ \\
\hline $\mathbf{3}$ & $\begin{array}{ll}\delta\left(\mathrm{AsCH}_{3}\right) & =0,7(\mathrm{~s}) \\
\delta\left(\mathrm{CH}_{2} \mathrm{OAs}\right) & =3,3(\mathrm{~m}) \\
\delta\left(\mathrm{CH}_{2} \mathrm{O}\right) & =2,9(\mathrm{~m}) \\
\delta\left(\mathrm{CH}_{3}\right) & =1,2(\mathrm{~m}) \\
\delta(\mathrm{OH}) & =3,6(\mathrm{~s}) \\
\text { Integration } & 12: 4: 2: 3: 1\end{array}$ & $\begin{array}{l}3380(\mathrm{~s}), 2980(\mathrm{~s}), 2930(\mathrm{ss}), 2890(\mathrm{~s}), 1480(\mathrm{~s}), 1430(\mathrm{ss}), 1310(\mathrm{w}) \\
1260(\mathrm{~m}), 1155(\mathrm{~m}), 1040(\mathrm{ss}), 970(\mathrm{~m}), 890(\mathrm{~s}), 825(\mathrm{~s}), 690(\mathrm{~s}) \\
580(\mathrm{ss})\end{array}$ \\
\hline 4 & $\begin{array}{ll}\delta\left(\mathrm{AsCH}_{3}\right) & =0,8(\mathrm{~s}) \\
\delta\left(\mathrm{CH}_{2} \mathrm{O}\right) & =3,8(\mathrm{~m}) \\
\delta\left(\mathrm{CH}_{3}\right) & =1,3(\mathrm{~m}) \\
\delta(\mathrm{OH}) & =3,6(\mathrm{~s}) \\
\text { Integration } & 3: 6: 3: 1\end{array}$ & $\begin{array}{l}3400(\mathrm{ss}), 2940(\mathrm{ss}), 2390(\mathrm{ss}), 1480(\mathrm{~s}), 1400(\mathrm{~s}), 1250(\mathrm{~s}), 1200(\mathrm{w}), \\
1160(\mathrm{~m}), 1030(\mathrm{ss}), 980(\mathrm{sh}), 915(\mathrm{~m}), 825(\mathrm{~s}), 690(\mathrm{ss}), 630(\mathrm{~s}), \\
585(\mathrm{ss}) .\end{array}$ \\
\hline $\mathbf{5}$ & $\begin{array}{l}\delta\left(\mathrm{AsCH}_{3}\right)=0,7(\mathrm{~s}) \\
\delta\left(\mathrm{OCH}_{2}\right)=3,5(\mathrm{~m}) \\
\delta\left(\mathrm{CH}_{3}\right)=1,3(\mathrm{~m}) \\
\text { Integration } 3: 2: 1\end{array}$ & $\begin{array}{l}3000(\mathrm{~s}), 2930(\mathrm{ss}), 2890(\mathrm{~s}), 1480(\mathrm{ss}), 1440(\mathrm{~s}), 1420(\mathrm{~s}), 1380(\mathrm{~s}) \\
1260(\mathrm{~s}), 1160(\mathrm{w}), 1050(\mathrm{ss}), 1015(\mathrm{ss}), 980(\mathrm{~s}), 895(\mathrm{~s}), 825(\mathrm{~s}) \\
700(\mathrm{ss}), 630(\mathrm{~m}), 590(\mathrm{ss})\end{array}$ \\
\hline
\end{tabular}


1 B. Englund, J. Prakt. Chem. 124, 191 [1930].

2 K. J. Kuz'min, Trudy Kazan. Khim. Tekhnol. Inst. im. S. M. Kirova 19-20, 217 [1954-55]; C. A. 51, $11245 \mathrm{i}[1957]$.

3 T. E. Stevens, J. Org. Chem. 24, 1715 [1959].

4 J. G. Verkade u. L. T. Reynolds, J. Org. Chem. $25,663[1960]$.

5 G. Kamai u. N. A. Chadaeva, Zh. Obshch. Khim. 32, 1130 [1962].

6 R. R. Shagidullin, N. A. Chadaeva, N. I. Zarubina u. G. Kamai, Izvst. Akad. Nauk SSSR, Ser. Khim. 1966, 1543 [1966].

7 Gulf Research and Development Company (J. F. Deffner), US-Pat. 3,621,044 (16. Nov. 1971); C. A. 76, 34405 v [1972].
8 F. Kober u. W. J. RühL, Z. Anorg. Allg. Chem., im Druck.

9 O. Adler u. F. Kober, Z. Anorg. Allg. Chem., im Druck.

10 T. Tzschach u. W. Lange, Z. Anorg. Allg. Chem. 326, 280 [1964].

11 O. J. Scherer u. M. Schmidt, Angew. Chem. 76, 787 [1964].

12 H. L. Long u. H. J. Emeléus, J. Chem. Soc. 1946, 1123 [1946].

13 H. J. Vetter u. H. Nöth, Z. Anorg. Allg. Chem. 330, 233 [1964]. 